\section{Clinical diagnostic value of telomere length measurement in inherited bone marrow failure syndromes}

Bone marrow failure (BMF) is characterized by a hypocellular marrow and encompasses a diverse group of inherited and acquired disorders. Inherited bone marrow failure syndromes (IBMFS) occur in approximately 5\%$30 \%$ of patients with BMF in pediatric cohorts and consist of more than 25 defined disease entities, including dyskeratosis congenita (DC), Fanconi anemia (FA), Diamond-Blackfan anemia (DBA), and ShwachmanDiamond syndrome (SDS). ${ }^{1}$ IBMFS are a heterogeneous group of disorders in which BMF is usually associated with physical abnormalities. The diagnosis of IBMFS previously relied on the recognition of characteristic clinical features. Recent diagnostic advances using next-generation sequencing have revealed that some patients initially diagnosed with idiopathic aplastic anemia (AA) had cryptic presentations of IBMFS. ${ }^{2}$ This issue is important as a more accurate diagnosis may improve treatment outcomes.

Telomeres are the end segments of chromosomes: they are composed of long DNA repeats and a protein complex, and are essential for genome integrity. Germline mutations in genes involved in telomere biology can result in significantly short telomere length (TL) in peripheral blood lymphocytes in patients with $\mathrm{DC} .^{3}$ Although there is a consensus on the usefulness of TL for screening for DC, but not for other IBMFS, several investigators have demonstrated that TL is excessively short in patients with $\mathrm{AA}^{4}$ and non-DC IBMFS, ${ }^{5}$ including FA, SDS, and DBA. To assess the diagnostic value of TL, we measured TL in 133 patients with BMF and compared it to that in patients with DC, non-DC IBMFS, and AA.

We retrospectively studied 133 patients (68 male and 65 female) with BMF in Japan between 2013 and 2018. We collected peripheral blood samples at diagnosis from all patients, measured TL from peripheral blood lymphocytes, and performed targeted sequencing analysis covering 184 genes associated with IBMFS (Online Supplementary Table S1), as described in our previous studies. ${ }^{2,4} \mathrm{TL}$ was measured by flow-fluorescence in situ hybridization (flow-FISH) using a Telomere PNA Kit (Dako Cytomation, Glostrup, Denmark) according to the manufacturer's instructions. We calculated the ageadjusted relative TL in terms of the standard deviation (SD) from 71 normal, age-matched, healthy controls (median age, 29 years; range, 1-47 years) as previously described. $^{4}$ In 112 of 133 (84\%) patients, paroxysmal nocturnal hemoglobinuria-type granulocytes and red blood cells were also evaluated by flow cytometry. ${ }^{4}$ As thresholds for determining the presence of minor paroxysmal nocturnal hemoglobinuria clones, we defined $>0.020 \%$ and $>0.037 \%$ for $\mathrm{CD} 11 \mathrm{~b}^{+} \mathrm{CD}^{-} 5^{-}$

Table 1. Clinical characteristics and laboratory findings of patients with bone marrow failure.

\begin{tabular}{|c|c|c|c|c|c|}
\hline & $\begin{array}{l}\text { All patients } \\
(\mathbb{N}=133)\end{array}$ & $\begin{array}{c}D C \\
(n=11)\end{array}$ & $\begin{array}{c}\text { Non-DC IBMFS } \\
(n=15)\end{array}$ & $\begin{array}{c}\text { AA } \\
(n=107)\end{array}$ & $P$ \\
\hline Age, years, median (range) & $7(0-22)$ & $7(1-19)$ & $6(0-15)$ & $7(0-22)$ & 0.675 \\
\hline \multicolumn{6}{|l|}{ Gender, n (\%) } \\
\hline Male & $68(51)$ & $5(45)$ & $7(47)$ & $56(52)$ & \multirow[t]{2}{*}{0.851} \\
\hline Female & $65(49)$ & $6(55)$ & $8(53)$ & $51(48)$ & \\
\hline \multicolumn{6}{|l|}{ Cytopenia, n (\%) } \\
\hline Unilineage cytopenia & $4(3)$ & $1(9)$ & $3(20)$ & $0(0)$ & \multirow[t]{3}{*}{$<0.001$} \\
\hline Bicytopenia & $24(18)$ & $3(27)$ & $3(20)$ & $18(17)$ & \\
\hline Pancytopenia & $105(79)$ & $7(64)$ & $9(60)$ & $89(83)$ & \\
\hline \multicolumn{6}{|l|}{ Severity, n (\%)* } \\
\hline Moderate & $67(52)$ & $7(70)$ & $10(83)$ & $50(47)$ & \multirow[t]{3}{*}{0.024} \\
\hline Severe & $35(27)$ & $2(20)$ & $2(17)$ & $31(29)$ & \\
\hline Very severe & $27(21)$ & $1(10)$ & $0(0)$ & $26(24)$ & \\
\hline WBC, $\times 10^{9} / \mathrm{L}$, median (range) & $2.8(0.3-12.8)$ & $2.7(1.7-5.3)$ & $3.1(1.7-7.8)$ & $2.8(0.3-12.8)$ & 0.374 \\
\hline ANC, ×109/L, median (range) & $0.6(0.0-5.4)$ & $0.8(0.6-1.7)$ & $0.9(0.2-5.4)$ & $0.5(0-5.2)$ & 0.011 \\
\hline ALC, $\times 10^{9} / \mathrm{L}$, median (range) & $1.8(0.1-8.2)$ & $1.3(0.5-4.3)$ & $2.1(1.2-5.9)$ & $1.8(0.1-8.2)$ & 0.065 \\
\hline $\mathrm{Hb}, \mathrm{g} / \mathrm{dL}$, median (range) & $8.0(2.7-14.3)$ & $8.4(3.1-14.3)$ & $6.6(3.7-11.3)$ & $8.1(2.7-14.0)$ & 0.307 \\
\hline Platelets, $\times 10^{9} / \mathrm{L}$, median (range) & $2.5(0.2-40.9)$ & $2.5(0.3-7.5)$ & $3.6(0.2-40.9)$ & $2.3(0.2-38.0)$ & 0.121 \\
\hline ARC, \%o, median (range) & $11.5(0.0-57.0)$ & $15.5(4.0-32.0)$ & $12.7(1.2-39.1)$ & $10.0(0.0-57.0)$ & 0.106 \\
\hline \multicolumn{6}{|l|}{ Minor PNH clones, n (\%) } \\
\hline Positive & $42(32)$ & $3(27)$ & $3(20)$ & $36(34)$ & \multirow[t]{3}{*}{0.632} \\
\hline Negative & $70(53)$ & $5(46)$ & $10(67)$ & $55(51)$ & \\
\hline Not done & $21(15)$ & $3(27)$ & $2(13)$ & $16(15)$ & \\
\hline Very short TL, <-2.19 SD, n (\%) & $31(23)$ & $10(91)$ & $4(25)$ & $17(16)$ & $<0.001$ \\
\hline Relatively short TL, <-1.71 SD, n (\%) & $44(33)$ & $10(91)$ & $9(60)$ & $25(23)$ & $<0.001$ \\
\hline TL, SD, median (range) & $0.96(-5.73$ to +4.00$)$ & $-3.50(-5.73$ to +0.83$)$ & $-1.89(-4.74$ to +2.05$)$ & $-0.84(-4.27$ to +4.00$)$ & $<0.001$ \\
\hline
\end{tabular}

DC: dyskeratosis congenital; IBMFS: inherited bone marrow failure syndromes; AA: aplastic anemia; WBC: white blood cell count; ANC: absolute neutrophil count; ALC: absolute lymphocyte count; Hb: hemoglobin; ARC: absolute reticulocyte count; PNH: paroxysmal nocturnal hemoglobinuria; TL: telomere length; SD: standard deviation; *excluding patients with unilineage cytopenia. 
A

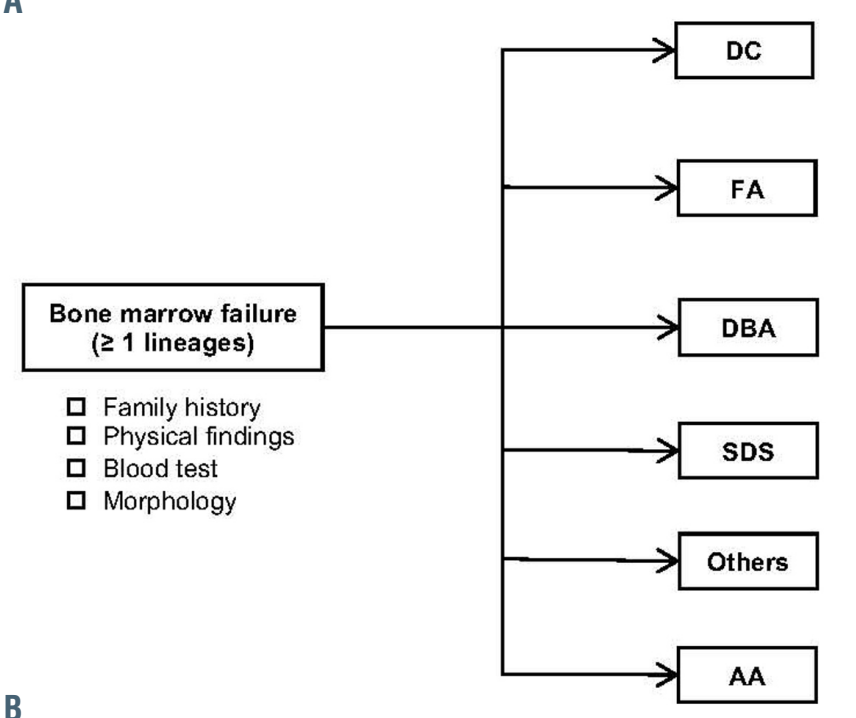

ㅁ Mucocutaneous triad of abnormalities

$\square$ Shortened telomere length

$\square$ Pathogenic germline mutation

C Chromosomal fragility test $(+)$

FANCD2 monoubiquitin test $(+)$

$\square$ Pathogenic germline mutation

Erythroid precursor $(\downarrow)$

Anemia from infancy

$\square$ Pathogenic germline mutation

$\square$ Exocrine pancreatic insufficiency

$\square$ Pathogenic germline mutation

$\square$ Pathogenic germline mutation

- Hypoplastic bone marrow ( $\geq 2$ lineages)

$\square$ Absence of underlying malignancy and IBMFS

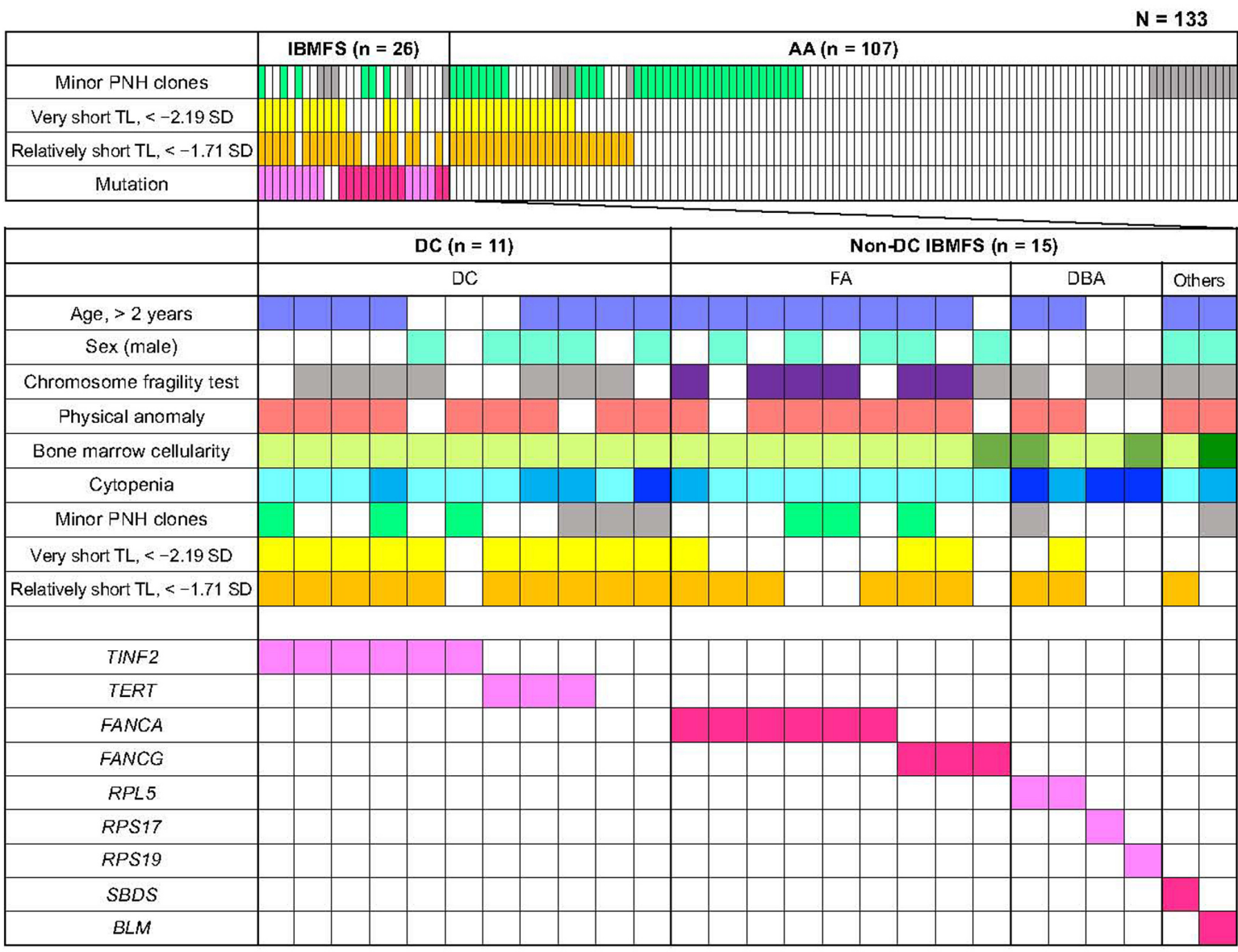

anor PNH clones

Not examined

Very short $\mathrm{TL},<-2.19 \mathrm{SD}$

Relatively short TL, $<-1.71 \mathrm{SD}$

Heterozygous mutation

Homozygous / Compound heterozygous mutation
Age, $>2$ years

Sex (male)

Chromosome fragility test

Physical anomaly
Hypocellular marrow

Normocellular marrow

Hypercellular marrow

- Pancytopenia

Bicytopenia

- Unilineage cytopenia

Figure 1. Diagnostic flowchart and profiles of patients with bone marrow failure. (A) Diagnostic flowchart for inherited bone marrow failure syndromes (IBMFS) and aplastic anemia (AA). The diagnoses were based on clinical criteria, syndrome-specific laboratory tests, and genetic analysis using targeted sequencing. (B) Clinical and genetic profiles of 133 patients with bone marrow failure (BMF). Each column indicates one patient. DC: dyskeratosis congenita; FA: Fanconi anemia; DBA: Diamond-Blackfan anemia; SDS: Shwachman-Diamond syndrome; AA: aplastic anemia; IBMFS: inherited bone marrow failure syndromes; PNH: paroxysmal nocturnal hemoglobinuria; TL: telomere length. 
$\mathrm{CD}^{+} 9^{+}$granulocytes and glycophorin $\mathrm{A}^{+} \mathrm{CD} 55^{-} \mathrm{CD} 59^{-}$ erythrocytes, respectively.

We diagnosed patients on the basis of a diagnostic flowchart (Figure 1A) developed using published diagnostic criteria for specific IBMFS and acquired AA. ${ }^{6,7}$ The severity of cytopenia was determined according to the Camitta severity criteria for AA. ${ }^{8}$ We divided the 133 patients into three groups: those with DC, those with non-DC IBMFS, and those with AA. All statistical analyses were performed using EZR (Saitama Medical Center, Jichi Medical University, Saitama, Japan). ${ }^{9}$ Written informed consent was obtained from patients or their legal guardians. This study was approved by the ethics committee of the Nagoya University Graduate School of Medicine.
Table 1 shows the clinical characteristics of patients included in this study. The median age at diagnosis of the total cohort was 7 years (range, $0-22$ years). Of the 133 patients, 105, 24, and 4 were diagnosed with pancytopenia, bicytopenia, and unilineage cytopenia ( 3 anemia and 1 thrombocytopenia), respectively. In patients with pancytopenia or bicytopenia, severity was assessed as very severe, severe, and moderate in 27,35 , and 67 patients, respectively. The median TL in all 133 patients was -0.96 $\mathrm{SD}$ (range, -5.73 to $+4.00 \mathrm{SD}$ ). Using targeted sequencing, in 24 patients $(18 \%)$ we detected 35 pathogenic variants $(5$ nonsense, 13 missense, 5 frameshift, 7 splice site, and 5 deletions) of known causative IBMFS genes, including TINF2 $(\mathrm{n}=6)$, TERT $(\mathrm{n}=3)$, FANCA $(\mathrm{n}=6)$,

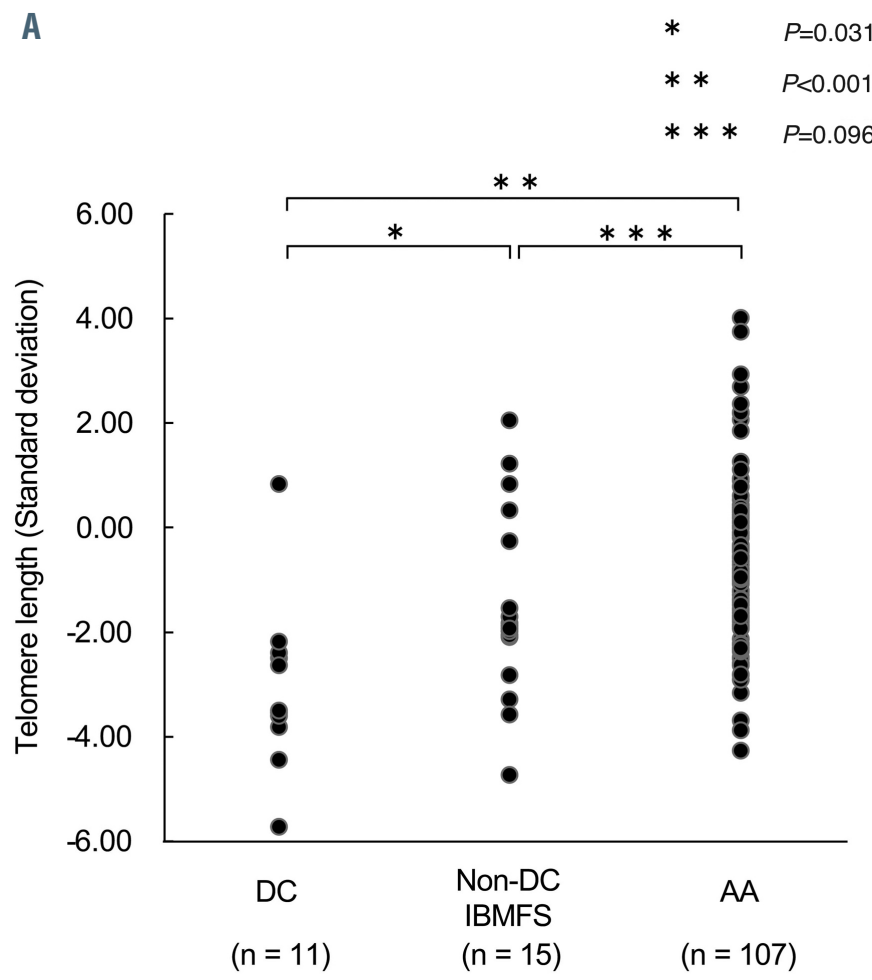

B
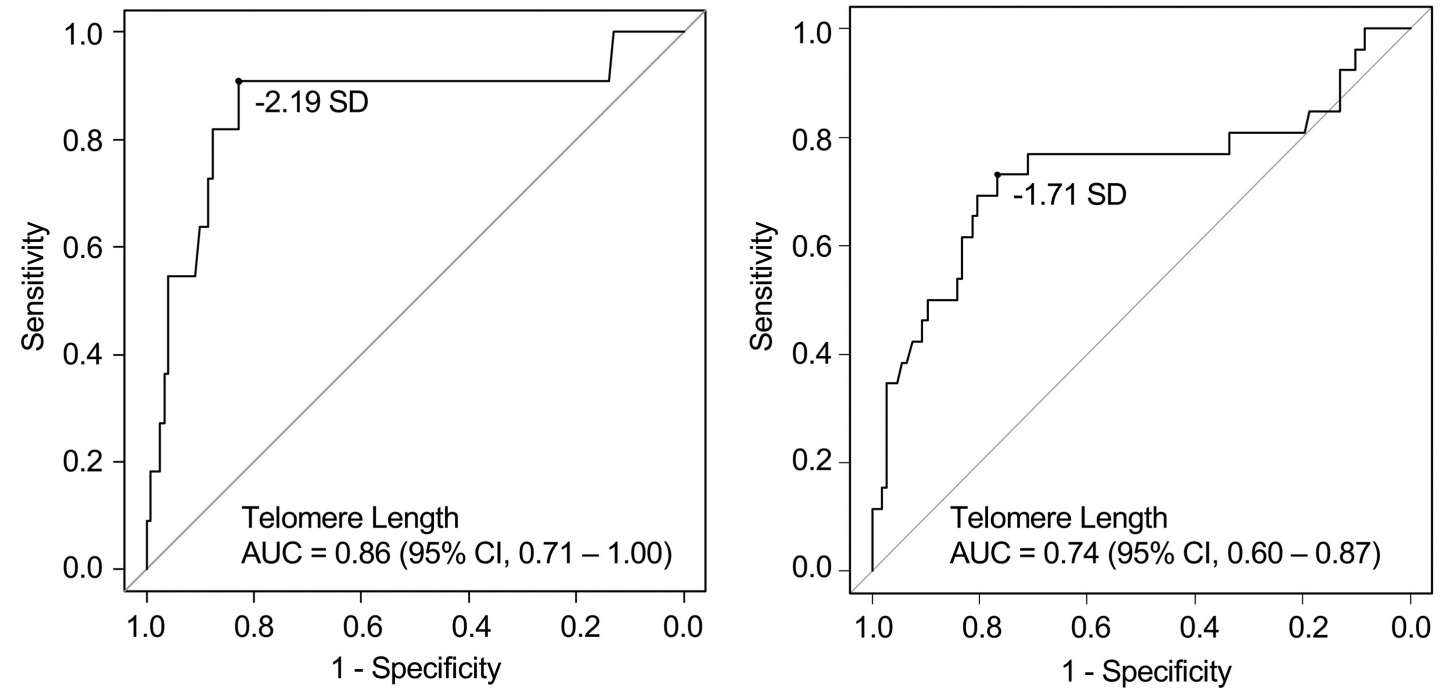
FANCG (n=3), RPL5 (n=2), RPS19 ( $\mathrm{n}=1), \operatorname{RPS} 17(\mathrm{n}=1)$, $\operatorname{SBDS}(\mathrm{n}=1)$, and $B L M(\mathrm{n}=1)$. Homozygous mutations were found in three patients (2 in FANCG and 1 in $F A N C A)$, compound heterozygous mutations in four patients ( 2 in FANCA and 1 each in FANCG and SBDS), hemizygous mutations in three patients in FANCA, and heterozygous mutations in 14 patients (6 in TINF2, 3 in TERT, 2 in RPL5, and 1 each in RPS17, RPS19, and BLM). Each patient's genetic variants are shown in Online Supplementary Table S2.

Out of the 133 patients and following the diagnostic flowchart (Figure 1A), 11 were diagnosed with DC (8\%), 15 with non-DC IBMFS (11\%), and 107 with AA (81\%). The schematic representation of the results of gene analysis and the clinical features of IBMFS are shown in Figure $1 \mathrm{~B}$. Of the 11 patients with $\mathrm{DC}$, nine were genetically diagnosed ( 6 with mutations in TINF2 and 3 with mutations in TERT), and those without diagnostic genetic mutations were diagnosed on the basis of clinical criteria. The 15 non-DC IBMFS cases consisted of nine FA, four DBA, one SDS, and one Bloom syndrome. All of these diagnoses were confirmed by the presence of germline mutations in IBMFS-related genes. Physical anomalies were observed in 11 of $15(73 \%)$ patients. The individual clinical features and genetic results of the patients with IBMFS are shown in Online Supplementary Table S2.

We compared the clinical characteristics of patients with DC, non-DC IBMFS, and AA (Table 1). The median age and gender distribution did not show significant differences among the three groups. Severe or very severe cytopenia was significantly more frequent $(P=0.024)$ in AA cases $(57 / 107,53 \%)$ than in $\mathrm{DC}(3 / 10,30 \%)$ and non-DC IBMFS cases (2/12, 17\%).

The median TL in patients with DC, non-DC IBMFS, and $\mathrm{AA}$ were $-3.50 \mathrm{SD}$ (range, -5.73 to $+0.83 \mathrm{SD}$ ), -1.89 $\mathrm{SD}$ (range, -4.74 to $+2.05 \mathrm{SD}$ ), and $-0.84 \mathrm{SD}$ (range, -4.27 to $+4.00 \mathrm{SD}$ ), respectively (Figure 2A). Patients with DC had significantly shorter TL compared to those with non-DC IBMFS $(P=0.031)$ and AA $(P<0.001)$. Furthermore, patients with non-DC IBMFS tended to have shorter TL than those with AA $(P=0.096)$.

To validate the efficacy of TL measurement in diagnosing DC and IBMFS, receiver operating characteristic curves identified two cut-off values with the optimum sensitivity and false positive rate (1-specificity) combination, <-2.19 SD (for patients with DC) (Figure 2B) and $<-1.71$ SD (for patients with IBMFS) (Figure 2C), defined as "very short TL" and "relatively short TL," respectively. For the diagnosis of patients with IBMFS, the TL cut-off value at $-1.71 \mathrm{SD}$ (relatively short TL) yielded a relatively high negative predictive value $(0.921 ; 95 \%$ confidence interval [95\% CI]: $0.873-0.958)$ and a moderately positive predictive value $(0.432 ; 95 \% \mathrm{CI}: 0.333-0.505)$. Of the total cohort, 44 patients (33\%) were classified as having "relatively short TL", which was significantly more frequent $(P<0.001)$ in DC $(10 / 11,91 \%)$ and non-DC IBMFS $(9 / 15,60 \%)$ than in AA $(25 / 107,23 \%)$.

Although germline mutations in TL maintenance genes are known to cause very short TL in patients with $\mathrm{DC},{ }^{3}$ several studies found that patients with AA also had shorter TL than healthy individuals. ${ }^{4}$ Furthermore, several cases of short TL in patients with non-DC IBMFS have been reported (Online Supplementary Table S3). ${ }^{3,5,10-15}$ Alter et al. ${ }^{5}$ previously reported that very short telomeres $\left(<1^{\text {st }}\right.$ percentile of normal) were observed in five of $78(6 \%)$ patients with non-DC IBMFS, although little overlap was seen in the distribution of TL between DC and non-DC IBMFS cases.
In this study we measured TL by standard flow-FISH in a cohort of BMF patients comprehensively and genetically evaluated by next-generation sequencing. We defined $\mathrm{TL}<-1.71 \mathrm{SD}$ of normal as a new criterion of "relatively short TL"; the proportions of patients who met this criterion were significantly higher in DC $(91 \%)$ and non-DC cases $(60 \%)$ than in AA cases $(23 \%)$. These results suggest that TL measurement is useful as a screening test for DC and as a clinical diagnostic tool for non-DC IBMFS patients needing comprehensive genetic analysis.

One limitation of this study is its small sample size: we found that $73 \%(n=26)$ of IBMFS cases and $23 \%(n=107)$ of AA cases in this cohort had "relatively short TL." A power calculation to check "relatively short TL" effectiveness in diagnosing IBMFS concluded that the power of 0.998 was high enough to support the assumption of a sufficient number of cases in this study.

A second limitation of the present study is the small number of non-DC cases $(n=15)$, which was insufficient to discuss the significance of TL measurements in each IBMFS subtype. However, "relatively short TL" (<-1.71 SD) was observed in six of nine FA cases, two of four DBA cases, and one case of SDS (Figure 1B). The median TL were -1.84 SD (range, -4.74 to $+2.05 \mathrm{SD}$ ), $-0.89 \mathrm{SD}$ (range, -2.83 to $+1.21 \mathrm{SD}$ ), and -1.99 for FA, DBA, and SDS cases, respectively (Online Supplementary Table S2). These results support those in previous case reports demonstrating relatively short TL in FA, DBA, and SDS (Online Supplementary Table S3). Nevertheless, future studies in larger cohorts of patients are warranted.

This study confirms that a relatively short TL was present in a significant proportion of patients with $\mathrm{DC}$ and non-DC IBMFS, indicating the clinical diagnostic value of TL measurement in identifying patients who need further testing, particularly comprehensive genetic analysis.

Shunsuke Miwata, Atsushi Narita, ${ }^{1}$ Yusuke Okuno, ${ }^{1,2}$ Kyogo Suzuki, ${ }^{1}$ Motoharu Hamada, ${ }^{1}$ Taro Yoshida, ${ }^{1}$ Masayuki Imaya, ${ }^{1}$ Ayako Yamamori, ${ }^{1}$ Manabu Wakamatsu, ${ }^{1}$

Kotaro Narita, ${ }^{1}$ Hironobu Kitazawa, ${ }^{1}$ Daisuke Ichikawa, ${ }^{1}$ Rieko Taniguchi, ${ }^{1}$ Nozomu Kawashima, ${ }^{1}$ Eri Nishikawa, ${ }^{1}$ Nobuhiro Nishio, ${ }^{1,2}$ Seiji Kojima, ${ }^{1}$ Hideki Muramatsu ${ }^{1}$ and Yoshiyuki Takahashi ${ }^{1}$

${ }^{1}$ Department of Pediatrics, Nagoya University Graduate School of Medicine and ${ }^{2}$ Center for Advanced Medicine and Clinical

Research, Nagoya University Hospital, Nagoya, Japan

Correspondence:

HIDEKI MURAMATSU - hideki-muramatsu@med.nagoya-u.ac.jp YOSHIYUKITAKAHASHI - ytakaha@med.nagoya-u.ac.jp doi:10.3324/haematol.2021.278334

Received: January 22, 2021

Accepted: April 13, 2021.

Pre-published: April 22, 2021

Disclosures: no conflicts of interest to disclose.

Contributions: SM, AN and HM performed laboratory analyses, gathered clinical information, designed and conducted the research, analyzed data, and wrote the paper; $M I, A Y, M W, K N, H K, D I$, $R T$ and $Y O$ performed laboratory analyses; $M H$ and $Y T$ gathered clinical information; $K S, N K, E N, N N$ and $S K$ conducted the research; YT directed the research and wrote the paper.

Acknowledgments: the authors acknowledge all the clinicians, patients, and their families involved in this study. The authors thank Ms. Yoshie Miura and Ms. Hiroko Ono for their valuable assistance. 


\section{References}

1. Chhabra P, Bhatia P, Singh M, et al. Pediatric bone marrow failure clinical, hematological and targeted next generation sequencing data. Blood Cells Mol Dis. 2021;87:102510.

2. Muramatsu H, Okuno Y, Yoshida K, et al. Clinical utility of nextgeneration sequencing for inherited bone marrow failure syndromes. Genet Med. 2017;19(7):796-802.

3. Du HY, Pumbo E, Ivanovich J, et al. TERC and TERT gene mutations in patients with bone marrow failure and the significance of telomere length measurements. Blood. 2009;113(2):309-316.

4. Sakaguchi H, Nishio N, Hama A, et al. Peripheral blood lymphocyte telomere length as a predictor of response to immunosuppressive therapy in childhood aplastic anemia. Haematologica. 2014; 99(8):1312-1316

5. Alter BP, Giri N, Savage SA, Rosenberg PS. Telomere length in inherited bone marrow failure syndromes. Haematologica. 2015;100(1):49-54

6. Marsh JCW, Ball SE, Cavenagh J, et al. Guidelines for the diagnosis and management of aplastic anaemia. $\mathrm{Br} \mathrm{J}$ Haematol. 2009;147(1):43-70

7. Shimamura A, Alter BP. Pathophysiology and management of inherited bone marrow failure syndromes. Blood Rev. 2010;24(3):101122.
8. Camitta BM, Storb R, Thomas ED. Aplastic anemia (second of two parts): pathogenesis, diagnosis, treatment, and prognosis. N Engl J Med. 1982;306(12):712-718.

9. Kanda Y. Investigation of the freely available easy-to-use software "EZR" for medical statistics. Bone Marrow Transplant. 2013;48(3):452-458.

10. Ball SE, Gibson FM, Rizzo S, Tooze JA, Marsh JCW, Gordon-Smith EC. Progressive telomere shortening in aplastic anemia. Blood. 1998;91(10):3582-3592.

11. Hanson H, Mathew CG, Docherty Z, Mackie Ogilvie C. Telomere shortening in Fanconi anaemia demonstrated by a direct FISH approach. Cytogen Cell Genet. 2001;93(3-4):203-206.

12. Thornley I, Dror Y, Sung L, Wynn RF, Freedman MH. Abnormal telomere shortening in leucocytes of children with ShwachmanDiamond syndrome. Br J Haematol. 2002;117(1):189-192.

13. Li X, Leteurtre F, Rocha V, et al. Abnormal telomere metabolism in Fanconi's anaemia correlates with genomic instability and the probability of developing severe aplastic anaemia. Br J Haematol. 2003; 120(5):836-845.

14. Pavesi E, Avondo F, Aspesi A, et al. Analysis of telomeres in peripheral blood cells from patients with bone marrow failure. Pediatr Blood Cancer. 2009;53(3):411-416.

15. Ong SY, Li ST, Wong GC, Ho AYL, Nagarajan C, Ngeow J. Delayed diagnosis of Shwachman Diamond syndrome with short telomeres and a review of cases in Asia. Leuk Res Rep. 2018;9:54-57. 